\title{
Tratamento de águas residuárias de suinocultura em reator anaeróbio operado em batelada sequencial
}

\author{
Treatment of swine wastewater in anaerobic sequencing batch reactor
}

\begin{abstract}
Roberto Alves de Oliveira
Engenheiro Agrônomo e Tecnólogo em Construção Civil pela Universidade Estadual Paulista (Unesp); Doutor em Engenharia Civil pela Escola de Engenharia de São Carlos da Universidade de São Paulo (USP); Professor assistente do Departamento de Engenharia Rural da Unesp
\end{abstract}

Rose Maria Duda

Engenheira Química pela Universidade Federal do Paraná (UFPR); Mestre em Microbiologia Agropecuária pela Unesp; Doutoranda em Microbiologia

Agropecuária pela Unesp

\begin{abstract}
Resumo
Neste estudo avaliou-se o desempenho de um reator anaeróbio operado em batelada sequencial, em escala piloto, com volume total de $280 \mathrm{~L}$, no tratamento de águas residuárias de suinocultura. As cargas orgânicas volumétricas aplicadas no reator foram de 4,42; 5,27; 9,33 e 11,79 g DQO total $(\mathrm{L}$ d)ำ . As eficiências médias de remoção de $\mathrm{DQO}_{\text {total' }}$ sólidos suspensos totais (SST) e sólidos suspensos voláteis (SSV) variaram de 56 a $87 \%$. O nitrogênio total Kjedahl (NTK), fósforo total (P-total) e magnésio (Mg) foram removidos com eficiências médias de 26 a 39\%. As produções volumétricas de metano variaram de 0,50 a 0,64 L $\mathrm{CH}_{4}(\mathrm{~L} \text { reator d })^{-1}$ e não foram observadas diferenças significativas. As relações sólidos voláteis/sólidos totais (SV/ST) do lodo de tal reator variaram de 0,74 a 0,58. As maiores concentrações médias de nutrientes no lodo do reator foram para o nitrogênio, fósforo, ferro e cálcio, com valores de 30.610 a $64.400,1.590$ a $9.870,6.180$ a 8.700 e 1.180 a $6.760 \mathrm{mg} \mathrm{kg}^{-1}$ base seca, respectivamente.
\end{abstract}

Palavras-chave: dejetos de suínos; digestão anaeróbia; metano; nutrientes; carga orgânica volumétrica.

\begin{abstract}
In the present study, we evaluated an anaerobic sequencing batch reactor, in pilot scale and with a total volume of $280 \mathrm{~L}$, for the treatment of swine wastewater. The organic loading rates applied in such reactor were 4.42; $5.27 ; 9.33$ and $11.79 \mathrm{~g} \mathrm{COD}_{\text {total }}(\mathrm{L} \mathrm{d})^{-1}$. The average efficiencies of removal of $\mathrm{COD}_{\text {total }}$ total solids suspension (TSS) and volatile suspension solids (VSS) varied from 56 to $87 \%$. The nutrients total Kjedahl nitrogen (TKN), total phosphorus (total P) and Mg

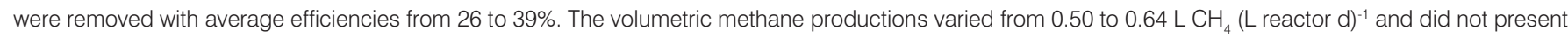
significant differences. The VS/TS relations of the aforementioned reactor's sludge varied from 0.74 to 0.58 . The highest mean concentrations of nutrients in the reactor sludge were those of nitrogen, phosphorus, iron and calcium, with values from 30.610 to $64.400,1.590$ to $9.870,6.180$ to 8.700 and 1.180 to 6.760 mg $\mathrm{kg}^{-1}$ base dry, respectively.
\end{abstract}

Keywords: swine wastewater; anaerobic digestion; methane, nutrients; organic loading rates.

\section{Introdução}

A suinocultura é uma das principais atividades agropecuárias que vem aumentando nas últimas décadas a sua participação nos impactos provocados no ambiente. A adoção de sistemas confinados de produção, com o uso intensivo da água para higienização das instalações, resulta em grandes quantidades de dejetos líquidos, com concentrações de sólidos de 0,5 a 3,0\% (FERNANDES; OLIVEIRA, 2006). Segundo a Pesquisa Agropecuária Municipal (IBGE, 2007) o plantel brasileiro de suínos é estimado em 35 milhões de cabeças, e o equivalente populacional médio em termos de $\mathrm{DBO}_{5,20}$ é de 3,5 habitantes por suíno (LINDNER, 1999, apud MIRANDA, 2005).

As águas residuárias de suinocultura contêm altos teores de matéria orgânica, nitrogênio, fósforo, potássio, cálcio, sódio, magnésio, manganês, ferro, zinco, cobre e outros elementos, incluídos nas dietas dos animais (LUDKE; LUDKE, 2002).

O tratamento biológico anaeróbio pode ser aplicado para resolver os problemas ambientais e de saúde pública associados às águas residuárias de suinocultura com altas cargas orgânicas, com as vantagens da produção de metano, baixa produção de lodo, facilidade na integração com o sistema de manejo de águas residuárias (NDON; 
DAGUE, 1997), conservação dos nutrientes e redução dos odores (AHN et al, 2006).

Como alternativa aos reatores anaeróbios de fluxo contínuo, houve o desenvolvimento do reator anaeróbio operado em batelada sequencial (ASBR, do inglês anaerobic sequencing batch reactor), com a realização de várias pesquisas que visam a sua aplicação prática no tratamento de resíduos sólidos e semissólidos (10 a 20\% de sólidos totais) e de águas residuárias (DAGUE et al, 1992; MASSE et al, 2003, 2007; NDON; DAGUE, 1997; SHIZAS; BAGLEY, 2002; ZHANG et al, 1997; MALTA-ALVAREZ, 2003). O ASBR tem algumas vantagens, como a eliminação da sedimentação secundária, boa retenção de biomassa, simplicidade de operação e flexibilidade (RODRIGUES et al, 2003; SHIZAS; BAGLEY, 2002). No ASBR, utiliza-se o processo anaeróbio de alta taxa para estabilizar águas residuárias, para controlar odores e produzir biogás como fonte alternativa de energia (NDON; DAGUE, 1997), além da possibilidade do uso agrícola do lodo excedente.

O tratamento biológico no ASBR é realizado em uma única unidade por meio de uma sequência operacional (ciclo), composta de quatro etapas distintas: alimentação, reação, sedimentação e descarte. A retenção de biomassa granular é um importante aspecto no ASBR e possibilita altas remoções de sólidos orgânicos, com baixos tempos de detenção hidráulica (NDON; DAGUE, 1997). Além dos aspectos físicos, os constituintes químicos e as propriedades da biomassa - que podem afetar a viabilidade da aplicação no solo do efluente e do lodo de forma benéfica - também devem ser conhecidos, e abrangem a matéria orgânica, os nutrientes, os micro-organismos patogênicos, os metais pesados e os componentes tóxicos (METCALF; EDDY, 2003).

Considerando-se as características do ASBR, foram realizados vários trabalhos com a sua aplicação para os resíduos da suinocultura. Zhang et al (1997) utilizaram um reator de 12 L, com a aplicação de

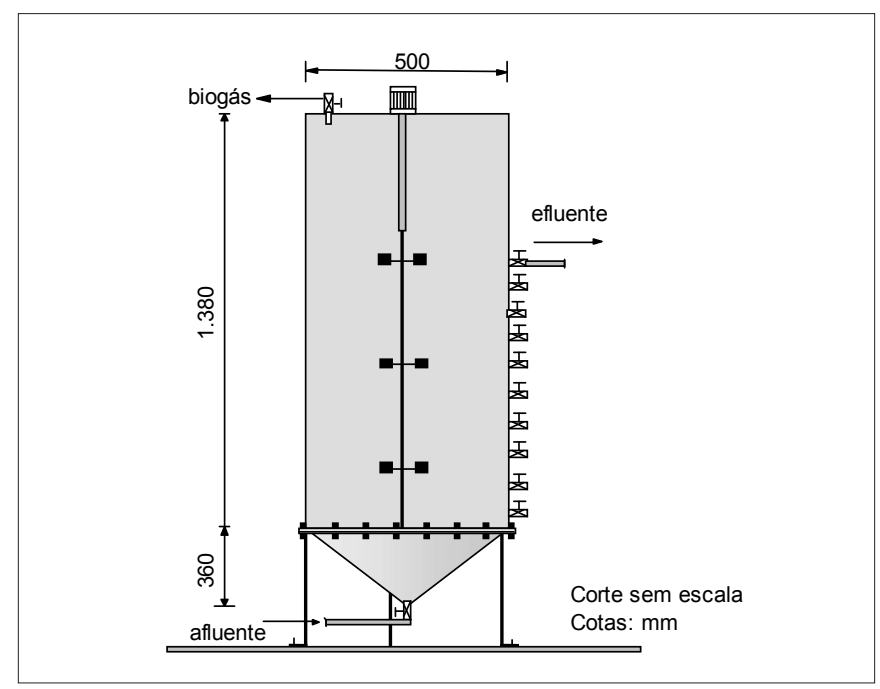

Figura 1 - Representação esquemática do reator ASBR em escala piloto, com volume de $280 \mathrm{~L}$ carga orgânica volumétrica (COV) de 0,9 a 5,5 g SV (L d)-1 e tempo de detenção hidráulica (TDH) de 2, 3 e 6 dias, com o afluente com sólidos voláteis (SV) de 2,7 a $17 \mathrm{~g} \mathrm{~L}^{-1}$, e obtiveram reduções de SV de 39 a $61 \%$, de $\mathrm{DBO}_{5}$ de 59 a $86 \%$ e produção volumétrica de biogás de 0,4 a 2,3 L ( $\mathrm{L}$ reator $\mathrm{d})^{-1}$, respectivamente. Masse et al (2003) trabalharam com reator de $42 \mathrm{~L}$, com COV de $1,2 \mathrm{~g} \mathrm{DQO}_{\text {total }}(\mathrm{L} \mathrm{d})^{-1}, \mathrm{TDH}$ de 28 dias e temperatura de $20^{\circ} \mathrm{C}$ no tratamento de afluente com $\mathrm{DQO}_{\text {total }}$ de $49 \mathrm{~g} \mathrm{~L}^{-1}$ e obtiveram eficiências de remoção de $\mathrm{DQO}_{\text {total }}$ de $89 \%$. Masse et al (2007) operam um reator de 41 L, com TDH de 4 semanas, $\mathrm{COV}$ de $4 \mathrm{~g} \mathrm{DQO}_{\text {total }}(\mathrm{L} \mathrm{d})^{-1}$ e temperatura de $20^{\circ} \mathrm{C}$, com afluente com $\mathrm{DQO}_{\text {total }}$ de $131 \mathrm{~g} \mathrm{~L}^{-1}$ e obtiveram eficiências médias de remoção de $\mathrm{DQO}_{\text {total }}$ de 58 a 73\%. A utilização do ASBR para a suinocultura com manejo de dejetos líquidos, em regiões sob clima tropical e subtropical depende do conhecimento da influência das condições operacionais (TDH e COV) e ambientais (temperatura) e das características do afluente e qualidade do efluente.

Portanto, neste trabalho foi avaliado o efeito do aumento da COV de 4,4 a 11,8 $\mathrm{g} \mathrm{DQO}_{\text {total }}(\mathrm{L} \mathrm{d})^{-1}$ com a variação da temperatura média do ar no desempenho de um ASBR, quanto à remoção de matéria orgânica, nutrientes, coliformes termotolerantes, produção de metano e características do lodo, no tratamento de águas residuárias de suinocultura, com concentrações de sólidos suspensos totais em torno de $10.000 \mathrm{mg} \mathrm{L}^{-1}$.

\section{Material e métodos}

\section{Local}

A unidade experimental utilizada para o tratamento das águas residuárias de suinocultura foi constituída por uma bomba centrífuga, um depósito de dejetos de suínos (afluente) e um ASBR em escala piloto. No local da instalação do ASBR, o clima, segundo a classificação de Koppen, é subtropical úmido, seco no inverno e com chuva no verão, com temperatura média anual de $21^{\circ} \mathrm{C}$.

\section{Instalações experimentais}

O ASBR foi construído com um tubo de PVC comercial, com diâmetro de $500 \mathrm{~mm}$ e com volume total de 280 L (Figura 1). O volume livre, reservado para o acúmulo de biogás, na parte superior do ASBR, foi de 14\% do volume total do reator (40 L). Para a monitorização da produção de biogás foram instalados gasômetros, conforme descrito por Fernandes e Oliveira (2006). No ASBR foi instalado um sistema de agitação mecânica, composto por três impelidores, eixo e moto-redutor, e temporizador para o acionamento intermitente. Durante a etapa de reação, o sistema de agitação mecânica foi acionado intermitentemente (15 minutos por hora), com velocidade de 20 a 25 rotações por minuto (rpm), resultando num gradiente de velocidade (G) em torno de $100 \mathrm{~s}^{-1}$. 


\section{Condições operacionais e afluente}

O experimento foi dividido em quatro ensaios, variando-se o TDH no ASBR, conforme descrito na Tabela 1.

Os dejetos de suínos utilizados como afluente no ASBR foram coletados diariamente no Setor de Suinocultura da Unesp, Jaboticabal (SP). Os dejetos foram coletados em confinamento de suínos, na fase de crescimento e terminação, com lâmina d'água nas baias. Após a coleta os dejetos de suínos foram peneirados (peneira com malha quadrada de $3 \mathrm{~mm}$ ) para retirada dos sólidos grosseiros.

O TDH foi calculado dividindo-se o volume útil do reator pelo volume de alimentação diário, conforme descrito por SHIZAS E BAGLEY (2002) e PINHEIRO et al (2008). A avaliação de desempenho da unidade experimental se baseou na monitorização da água residuária de suinocultura bruta afluente e do efluente tratado no
ASBR, em amostras simples, perfazendo 15 amostragens para cada TDH aplicado.

\section{Delineamento experimental, exames e determinações}

O delineamento experimental utilizado foi o inteiramente casualizado, com quatro tratamentos (TDH de 96, 72, 48 e 36 horas) e 15 repetições para o afluente e efluente, 55 repetições para a produção volumétrica de metano e temperatura média do ar e oito repetições para sólidos totais e voláteis do lodo. Os valores dos parâmetros determinados, descritos na Tabela 2, foram submetidos à análise de variância pelo teste F, utilizando-se para a comparação das médias, o teste de Tukey a $5 \%$. As repetições foram as amostragens no tempo. Às médias das eficiências de remoção foi aplicada análise de regressão polinomial.

Tabela 1 - Distribuição dos tempos do ciclo operacional e volumes de afluente para cada condição operacional do ASBR

\begin{tabular}{|c|c|c|c|c|}
\hline Características & Ensaio 1 & Ensaio 2 & Ensaio 3 & Ensaio 4 \\
\hline Volume de alimentação diário (L) & 60 & 80 & 120 & 160 \\
\hline Tempo de detenção hidráulica (h) & 96 & 72 & 48 & 36 \\
\hline Tempo do ciclo (h) & 24 & 24 & 12 & 12 \\
\hline Tempo de alimentação (h) & 0,17 & 0,17 & 0,17 & 0,17 \\
\hline Tempo de reação $(\mathrm{h})$ & 19,00 & 19,00 & 9,00 & 9,00 \\
\hline Tempo de sedimentação (h) & 4,66 & 4,66 & 2,66 & 2,66 \\
\hline Tempo de retirada do sobrenadante (h) & 0,17 & 0,17 & 0,17 & 0,17 \\
\hline
\end{tabular}

Tabela 2 - Exames e determinações, frequência e fontes das metodologias utilizadas

\begin{tabular}{|c|c|c|}
\hline Exames e determinações & Frequência & Referências \\
\hline \multicolumn{3}{|l|}{ Afluente e efluentes } \\
\hline Temperatura média do ar & diária & Unesp, Jaboticabal - Estação Agroclimatológica \\
\hline $\mathrm{pH}$ e alcalinidade total (AT) & duas vezes por semana & APHA, AWWA, WPCF (1998) \\
\hline Ácidos voláteis totais & duas vezes por semana & Dilallo e Albertson (1961) \\
\hline Sólidos suspensos totais (SST) e voláteis (SSV) & duas vezes por semana & APHA, AWWA, WPCF (1998) \\
\hline $\begin{array}{l}\text { Demanda química de oxigênio }\left(\mathrm{DQO}_{\text {total }}\right) \text {; DQO } \\
\text { das frações dissolvida }\left(\mathrm{DQO}_{\text {diss. }}\right) \text { e de sólidos } \\
\text { suspensos }\left(\mathrm{DQO}_{\mathrm{ss}}\right)\end{array}$ & duas vezes por semana & $\begin{array}{l}\text { APHA, AWWA, WPCF (1998); Santana e Oliveira } \\
\text { (2005) (digestão com refluxo fechado e método } \\
\text { colorimétrico). }\end{array}$ \\
\hline Nitrogênio total Kjedahl (NTK) & duas vezes por semana & $\begin{array}{l}\text { APHA, AWWA, WPCF (1998) (obtenção } \\
\text { do extrato utilizando-se a digestão com } \\
\text { ácido sulfúrico e peróxido de hidrogênio no } \\
\text { equipamento Digesdahl, conforme instruções do } \\
\text { fabricante-Hach e posterior utilização do método } \\
\text { semimicro Kjedahl). }\end{array}$ \\
\hline $\begin{array}{l}\text { Nitrogênio amoniacal (N-am.) e Nitrogênio } \\
\text { orgânico (N-org. = NTK - N-am) }\end{array}$ & duas vezes por semana & $\begin{array}{l}\text { APHA, AWWA, WPCF (1998) (método semi- } \\
\text { micro Kjedahl). }\end{array}$ \\
\hline Fósforo total (P total) & duas vezes por semana & $\begin{array}{l}\text { APHA, AWWA, WPCF (1998) (obtenção do } \\
\text { extrato conforme descrito para o NTK e posterior } \\
\text { utilização do método colorimétrico empregando } \\
\text { metavanadato e molibdato de amônio). }\end{array}$ \\
\hline $\begin{array}{l}\text { Potássio, cálcio, magnésio, cobre, ferro, } \\
\text { manganês, sódio e zinco }\end{array}$ & duas vezes por semana & $\begin{array}{l}\text { APHA, AWWA, WPCF (1998) (obtenção do extrato } \\
\text { conforme descrito para o NTK e posterior leitura } \\
\text { em espectrofotômetro de absorção atômica). }\end{array}$ \\
\hline Coliformes totais e termotolerantes & duas vezes por ensaio & $\begin{array}{l}\text { APHA, AWWA, WPCF (1998) (técnica dos tubos } \\
\text { múltiplos) }\end{array}$ \\
\hline \multicolumn{3}{|l|}{ Biogás } \\
\hline Produção & diária & Oliveira (1997) (gasômetros) \\
\hline Composição & quinzenal & $\begin{array}{l}\text { APHA, AWWA, WPCF (1998) (cromatografia } \\
\text { gasosa). }\end{array}$ \\
\hline \multicolumn{3}{|l|}{ Lodo } \\
\hline Sólidos totais (ST) e voláteis (SV) & semanal & APHA, AWWA, WPCF (1998) \\
\hline $\begin{array}{l}\text { Nitrogênio total, fósforo total, potássio, cálcio, } \\
\text { magnésio, cobre, ferro, manganês, níquel, sódio } \\
\text { e zinco }\end{array}$ & uma vez por ensaio & $\begin{array}{l}\text { APHA, AWWA, WPCF (1998) (idem aos métodos } \\
\text { descritos para as determinações no afluente e } \\
\text { efluentes. }\end{array}$ \\
\hline
\end{tabular}




\section{Resultados e discussão}

$\mathrm{Na}$ Tabela 3, observa-se que, entre as temperaturas médias do ar e as COV aplicadas no ASBR, ocorreram diferenças significativas. As maiores e menores temperaturas climatológicas médias de $24,1^{\circ} \mathrm{C}$ e de $19,2^{\circ} \mathrm{C}$ foram observadas com os TDH de 96 e 48 horas, respectivamente.
Para os valores médios de $\mathrm{pH}$ (de 5,7 a 6,0) (Tabela 3), $\mathrm{DQO}_{\text {total }}$ (de 15.835 a $18.848 \mathrm{mg} \mathrm{L}^{-1}$ ), DQO (de 13.490 a $15.264 \mathrm{mg} \mathrm{L}^{-1}$ ) e SST (de 9.219 a $10.696 \mathrm{mg} \mathrm{L}^{-1}$ ) do afluente e da produção volumétrica de metano (de 0,50 a $0,64 \mathrm{~m}^{3} \mathrm{CH}_{4}\left(\mathrm{~m}^{3}\right.$ reator $\left.\mathrm{d}^{-1}\right)$ ) do ASBR (Tabela 4), não foram observadas diferenças significativas ( $p>0,05)$.

Os valores médios da $\mathrm{DQO}_{\text {total }}$ (de 2.756 a $\left.8.038 \mathrm{mg} \mathrm{L}^{-1}\right), \mathrm{DQO}_{\text {diss }}$ (de 508 a $\left.1.758 \mathrm{mg} \mathrm{L}^{-1}\right), \mathrm{DQO}_{\mathrm{sS}}\left(\mathrm{de} 3.197\right.$ a $\left.6.280 \mathrm{mg} \mathrm{L}^{-1}\right)$, SST

Tabela 3 - Valores médios do pH, da temperatura média do ar, AT e AVT no afluente e efluente do ASBR durante os ensaios

\begin{tabular}{|c|c|c|c|c|c|c|c|c|c|c|c|}
\hline \multirow{3}{*}{$\begin{array}{l}\text { Parâmetros } \\
\text { Temperatura }(\stackrel{\circ}{ } \mathrm{C})\end{array}$} & \multirow[b]{3}{*}{ - } & \multicolumn{8}{|c|}{ Tempo de detenção hidráulico (h) } & \multirow{3}{*}{$\begin{array}{c}\text { CV (\%) } \\
9,3\end{array}$} & \multirow{3}{*}{$\begin{array}{l}\text { Teste F } \\
47,2^{* *}\end{array}$} \\
\hline & & \multicolumn{2}{|c|}{96} & \multicolumn{2}{|c|}{72} & \multicolumn{2}{|c|}{48} & \multicolumn{2}{|c|}{36} & & \\
\hline & & 24,1 & a & 22,5 & $b$ & 19,2 & $c$ & 22,4 & $b$ & & \\
\hline $\begin{array}{l}\text { COV } \\
\left(\mathrm{g} \mathrm{DQO} \mathrm{total}(\mathrm{L} \mathrm{d})^{-1}\right)\end{array}$ & - & 4,42 & $\mathrm{~b}$ & 5,27 & $\mathrm{~b}$ & 9,33 & a & 11,79 & a & 36,7 & 22,4 ** \\
\hline \multirow{2}{*}{$\mathrm{pH}$} & Afluente & 5,7 & a & 5,8 & a & 5,9 & a & 6,0 & a & 7,6 & $1,5 \mathrm{~ns}$ \\
\hline & Efluente & 7,4 & a & 7,2 & $a b$ & 6,9 & $\mathrm{~b}$ & 7,4 & $\mathrm{a}$ & 4,7 & $6,6^{\star \star}$ \\
\hline \multirow{2}{*}{$\begin{array}{l}\text { AT } \\
\left(\mathrm{mg} \mathrm{CaCO}_{3} \mathrm{~L}^{-1}\right)\end{array}$} & Afluente & 1.358 & $a$ & 909 & $b$ & 1.132 & $a b$ & 1.342 & $a$ & 35,7 & $3,7^{*}$ \\
\hline & Efluente & 2.090 & $a$ & 1.996 & $\mathrm{c}$ & 1.641 & bc & 2.075 & $a b$ & 24,7 & $5,2^{\star \star}$ \\
\hline \multirow{2}{*}{$\begin{array}{l}\text { AVT } \\
\left(\mathrm{mg} \mathrm{CH}_{3} \mathrm{COOH} \mathrm{L}^{-1}\right)\end{array}$} & Afluente & 1.465 & $a$ & 919 & $b$ & 1.134 & $a b$ & 1.321 & $a b$ & 35,1 & $4,65^{\star \star}$ \\
\hline & Efluente & 357 & $b$ & 194 & $c$ & 509 & $\mathrm{a}$ & 226 & bc & 47,5 & $13,1^{\star \star}$ \\
\hline
\end{tabular}

Letras minúsculas diferentes na mesma linha diferem pelo teste de Tukey a $5 \%$; ** significativo a $1 \%$ de probabilidade $(p<0,01)$; * significativo a $5 \%$ de probabilidade ( $p<0,05)$; ns: nãosignificativo ( $p>0,05)$; $p$ : probabilidade; COV: carga orgânica volumétrica; AT: alcalinidade total; AVT: ácidos voláteis totais.

Tabela 4 - Valores médios $\mathrm{DQO}_{\text {total, }}, \mathrm{DQO}_{\text {diss, }}, \mathrm{DQO}_{\mathrm{ss}}$, SST, SSV e dos coliformes termotolerantes no afluente e efluente; das eficiências de remoção de $\mathrm{DQO}_{\text {total }}, \mathrm{DQ0_{ \text {diss } }}, \mathrm{DQO_{ \text {ss } }}, \mathrm{SST}$ e SSV e da volumétrica de metano do ASBR durante os ensaios

\begin{tabular}{|c|c|c|c|c|c|c|c|c|c|c|c|}
\hline \multirow{3}{*}{$\begin{array}{l}\text { Parâmetros } \\
\mathrm{DQO}_{\text {total }}\left(\mathrm{mg} \mathrm{L}^{-1}\right)\end{array}$} & \multirow[b]{3}{*}{ Afluente } & \multicolumn{8}{|c|}{ Tempo de detenção hidráulica (h) } & \multirow{3}{*}{$\begin{array}{c}\text { CV (\%) } \\
31,8\end{array}$} & \multirow{3}{*}{$\begin{array}{l}\text { Teste F } \\
0,89 \text { ns }\end{array}$} \\
\hline & & \multicolumn{2}{|c|}{96} & \multicolumn{2}{|c|}{72} & \multicolumn{2}{|c|}{48} & \multicolumn{2}{|c|}{36} & & \\
\hline & & 17.727 & a & 15.835 & a & 18.674 & a & 18.848 & a & & \\
\hline & Efluente & 2.756 & $\mathrm{~b}$ & 3.705 & $\mathrm{~b}$ & 8.038 & a & 4.415 & $b$ & 65,4 & $8,3^{\star \star}$ \\
\hline & Eficiência (\%) & 84 & a & 68 & $a b$ & 56 & $\mathrm{~b}$ & 75 & a & 27,0 & $5,6^{\star \star}$ \\
\hline \multirow[t]{3}{*}{$\mathrm{DQO}_{\text {diss }}\left(\mathrm{mg} \mathrm{L}^{-1}\right)$} & Afluente & 2.632 & $\mathrm{~b}$ & 2.345 & $\mathrm{~b}$ & 3.410 & $a b$ & 3.977 & a & 37,5 & $6,2^{\star *}$ \\
\hline & Efluente & 888 & $b$ & 508 & $b$ & 1.758 & a & 989 & $\mathrm{~b}$ & 50,3 & $15,1^{\star \star}$ \\
\hline & Eficiência (\%) & 63 & $a b$ & 78 & $\mathrm{a}$ & 48 & $b$ & 75 & a & 25,8 & $9,3^{* *}$ \\
\hline \multirow[t]{3}{*}{$\mathrm{DQO}_{\mathrm{ss}}\left(\mathrm{mg} \mathrm{L}^{-1}\right)$} & Afluente & 14.892 & a & 13.490 & $\mathrm{a}$ & 15.264 & a & 14.859 & a & 34,8 & $0,35 \mathrm{~ns}$ \\
\hline & Efluente & 1.868 & $b$ & 3.197 & $b$ & 6.280 & a & 3.426 & $a b$ & 82,2 & $5,6^{\star \star}$ \\
\hline & Eficiência (\%) & 87 & $a$ & 71 & $a b$ & 58 & $b$ & 77 & $a b$ & 29,1 & $4,8^{\star \star}$ \\
\hline \multirow[t]{3}{*}{$\mathrm{SST}\left(\mathrm{mg} \mathrm{L}^{-1}\right)$} & Afluente & 10.696 & a & 9.967 & $\mathrm{a}$ & 9.406 & a & 9.219 & a & 32,1 & $0,6 \mathrm{~ns}$ \\
\hline & Efluente & 1.553 & $\mathrm{~b}$ & 1.611 & $\mathrm{~b}$ & 3.954 & a & 1.985 & $\mathrm{~b}$ & 68,2 & $8,0^{\star *}$ \\
\hline & Eficiência (\%) & 85 & a & 82 & $\mathrm{a}$ & 56 & $b$ & 75 & a & 24,0 & $7,7^{\star \star}$ \\
\hline \multirow[t]{3}{*}{$\mathrm{SSV}\left(\mathrm{mg} \mathrm{L}^{-1}\right)$} & Afluente & 7.459 & $\mathrm{a}$ & 5.247 & $a b$ & 5.713 & $a b$ & 4.978 & $\mathrm{~b}$ & 40,6 & $3,3^{*}$ \\
\hline & Efluente & 1.157 & $b$ & 910 & $b$ & 2.332 & a & 1.157 & $\mathrm{~b}$ & 66,5 & $7,1^{\star \star}$ \\
\hline & Eficiência (\%) & 84 & $a$ & 80 & $a$ & 59 & $\mathrm{~b}$ & 73 & $a b$ & 24,1 & $5,6^{\star \star}$ \\
\hline $\begin{array}{l}\text { Produção } \\
\text { volumétrica de } \\
\text { metano }\left(\mathrm{LCH}_{4}\right. \\
\left.\left(\mathrm{L} \text { reator } \mathrm{CH}_{4} \mathrm{~d}\right)^{-1}\right)\end{array}$ & & 0,534 & a & 0,64 & $\mathrm{a}$ & 0,538 & a & 0,5 & a & 31,9 & $1,7 \mathrm{~ns}$ \\
\hline \multirow{3}{*}{$\begin{array}{l}\text { Coliformes } \\
\text { termotolerantes } \\
\text { (NMP/100 mL) }\end{array}$} & Afluente & $1,4 \times 10^{7}$ & & \multicolumn{2}{|c|}{$4,0 \times 10^{7}$} & \multicolumn{2}{|c|}{$1,6 \times 10^{8}$} & \multicolumn{2}{|c|}{$7,4 \times 10^{6}$} & - & - \\
\hline & Efluente & $9,0 \times 10^{4}$ & & \multicolumn{2}{|c|}{$2,5 \times 10^{6}$} & \multicolumn{2}{|c|}{$1,3 \times 10^{7}$} & \multicolumn{2}{|c|}{$7,5 \times 10^{5}$} & - & - \\
\hline & Eficiência (\%) & 99,35 & & \multicolumn{2}{|c|}{93,75} & \multicolumn{2}{|c|}{91,87} & \multicolumn{2}{|c|}{89,86} & - & - \\
\hline
\end{tabular}

Letras minúsculas diferentes na mesma linha diferem pelo teste de Tukey a $5 \%$; ** significativo a $1 \%$ de probabilidade $(p<0,01)$; * significativo a $5 \%$ de probabilidade ( $p<0,05)$; ns: nãosignificativo ( $p>0,05)$; $p$ : probabilidade; $\mathrm{DQO}_{\text {total }}$ : demanda química de oxigênio total; $\mathrm{DQO}_{\text {diss: }}$ : demanda química de oxigênio dissolvida; $\mathrm{DQO}$ : demanda química de oxigênio devido aos sólidos suspensos; SST: sólidos suspensos totais; SSV: sólidos suspensos voláteis (SSV). 
(de 1.553 a $3.954 \mathrm{mg} \mathrm{L}^{-1}$ ) e SSV (de 910 a $2.332 \mathrm{mg} \mathrm{L}^{-1}$ ) no efluente diminuíram acentuadamente $(\mathrm{p}<0,01)$, indicando o efeito da variação do TDH e da temperatura climatológica no desempenho do ASBR para a remoção de matéria orgânica (Tabela 4).

$\mathrm{Na}$ Tabela 4 e na Figura 2, pelas linhas de tendência, observase que as maiores eficiências de remoção de $\mathrm{DQO}_{\text {total }}$, de 84 e $75 \%$, ocorreram com os TDH de 96 e 36 horas, respectivamente, indicando resposta quadrática. As variações das eficiências de remoção de $\mathrm{DQO}_{\text {ss }}$ foram semelhantes às observadas para a $\mathrm{DQO}_{\text {total }}$, em razão dos altos níveis de $\mathrm{DQO}_{\mathrm{ss}}$ na composição da $\mathrm{DQO}_{\text {total }}$. Para a $\mathrm{DQO}_{\text {diss' }}$, as maiores eficiências de remoção de 78 e $75 \%$, foram verificadas com o TDH de 72 e 36 horas, respectivamente. Os valores obtidos para a $\mathrm{DQO}_{\text {total }}$ nos ensaios com TDH de 72, 48 e 36 horas, quando ocorreram as temperaturas médias do ar de 22,$5 ; 19,2$ e $22,4^{\circ} \mathrm{C}$ foram similares às observadas por Masse et al (2007), mesmo com TDH até 19 vezes inferiores.

Para as eficiências médias de remoção de $\mathrm{DQO}_{\text {total }}$, $\mathrm{DQO}_{\text {diss, }}$, $\mathrm{DQO}_{\mathrm{ss}}$, SST e SSV, não foram observadas diferenças significativas ( $\mathrm{p}$ $<0,01$ ) (Tabela 4), e os menores valores de 56; 48; 58, 56 e 59\% (p $<0,05)$, respectivamente, foram provocados pela menor temperatura média do ar de $19,2^{\circ} \mathrm{C}$, que ocorreu durante a operação com o TDH de 48 horas. Segundo Masse et al (1997), o decréscimo da temperatura diminui a atividade dos microrganismos anaeróbios, afetando o desempenho do ASBR para a remoção de matéria orgânica e dos sólidos suspensos.

Na Figura 3, pelas linhas de tendência, observa-se que ocorreu uma resposta linear para as eficiências de remoção dos SST e dos SSV, ou seja, com o aumento do TDH houve aumento na eficiência de remoção de SST e de SSV.

Os valores médios dos números de coliformes termotolerantes no afluente variaram de 7,4 x $10^{6}$ a 1,6 x $10^{8} \mathrm{NMP} / 100 \mathrm{~mL}$. No efluente, os coliformes termotolerantes variaram de $9,0 \times 10^{4}$ a $1,3 \times 10^{7}$ NMP/100 mL (Tabela 4).

No ASBR com o aumento do TDH de 36 para 96 horas e o decréscimo da COV de 11,79 para 4,42 g DQOtotal (L d) ${ }^{-1}$, foi observado aumento nas eficiências médias de remoção de coliformes termotolerantes de 89,86 para 99,35\%. As concentrações nos efluentes mantiveram-se acima de 9,0 × $10^{4} \mathrm{NMP} / 100 \mathrm{~mL}$, restringindo a aplicação das águas residuárias tratadas para a irrigação de plantas, tomando-se por base os valores estabelecidos nas diretrizes da Organização Mundial da Saúde $(1989,2006)$ e na resolução no 357 de 17 de março de 2005 do Conama (BRASIL, 2005), na qual estão definidos os limites de coliformes termotolerantes de 200, 1000 e 4000 NMP/100 mL em corpos d'água doce de classe 1, 2 e 3, dos quais podem ser captadas águas para irrigação.

Durante os ensaios, os valores médios de NTK, N-am., N-org. e P-total no afluente variaram de 752 a $1116 \mathrm{mg} \mathrm{L}^{-1}, 164$ a $274 \mathrm{mg} \mathrm{L}^{-1}$; 631 a $858 \mathrm{mg} \mathrm{L}^{-1}$ e de 338 a $448 \mathrm{mg} \mathrm{L}^{-1}$, respectivamente (Tabela 5). Costa E Medri (2002) observaram concentrações de NTK e P-total

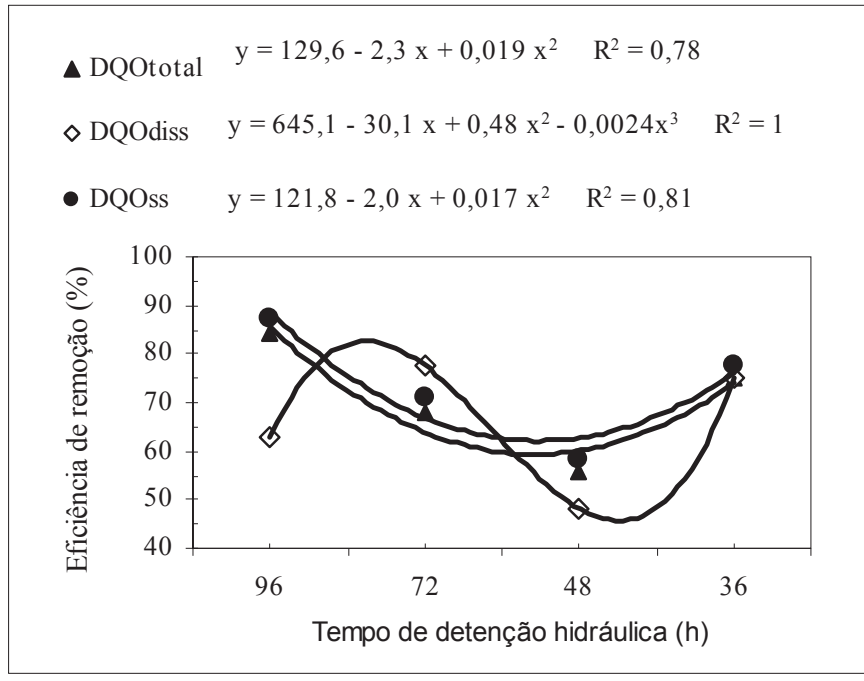

Figura 2 - Tempo de detenção hidráulica (TDH) versus eficiências de remoção (\%) de $\mathrm{DQO} \mathrm{total}_{\text {, }}$ DQO ${ }_{\text {diss }}$ e $\mathrm{DQO} \mathrm{O}_{\mathrm{ss}}$ no $\mathrm{ASBR}$

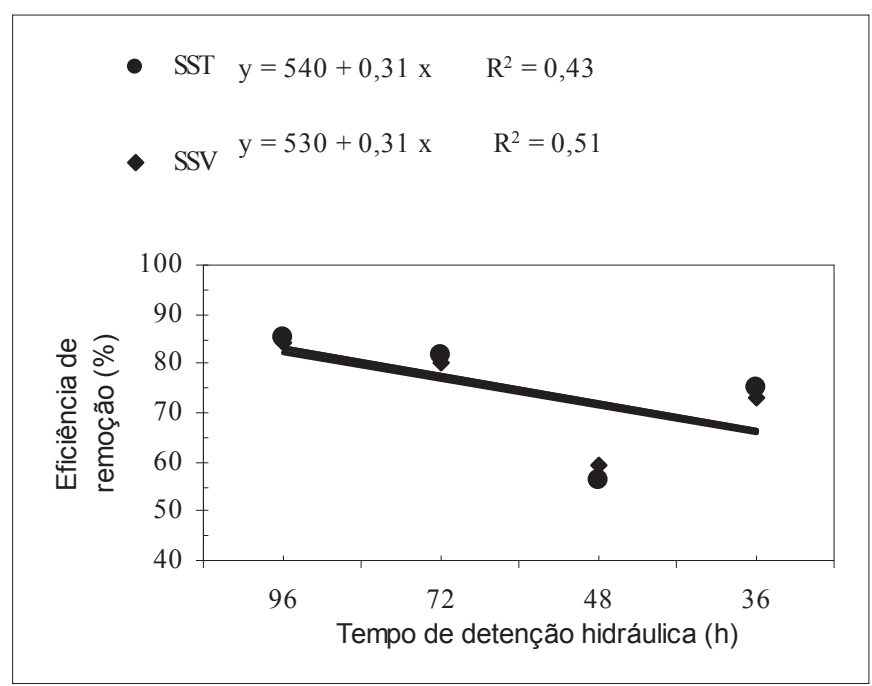

Figura 3 - Tempo de detenção hidráulica (TDH) versus eficiências de remoção (\%) de SST e SSV no ASBR

próximas às observadas neste trabalho, de $1.825 \mathrm{mg} \mathrm{L}^{-1} \mathrm{e} 391 \mathrm{mg} \mathrm{L}^{-1}$, respectivamente, em águas residuárias de suinocultura com $\mathrm{DQO}_{\text {total }}$ de $15.153 \mathrm{mg} \mathrm{L}^{-1}$.

As eficiências médias de remoção do ASBR foram de 31 a 39\% para o NTK, 59 a $80 \%$ para o N-org. e de 32 a 38\% para o P-total. Observase que as menores eficiências de remoção de N-org. de 59\% e 65\% ocorreram quando se aplicaram as maiores COV de 11,79 e 9,33 g DQO ( $(\mathrm{d})^{-1}$, e com as menores temperaturas, provocando decréscimo na digestão de material proteico (Tabelas 3 e 5). As eficiências de remoção de P-total observadas neste estudo foram superiores às observadas por MASSE et al (2007), de 25,5\%; tratando águas residuárias de suinocultura em um ASBR com COV de 0,14 g DQO total $(\mathrm{L} \mathrm{d})^{-1}$.

No ASBR observaram-se eficiências de remoção de 45 a 63\% para o cálcio (Ca). Não foram observadas remoções de sódio $(\mathrm{Na})$ e potássio (K) (Tabela 6). 
Tabela 5 - Valores médios das COV aplicadas no ASBR e das concentrações e eficiências médias de remoção de NTK, N-am., N-org. e P-total do afluente e efluentes do ASBR durante os ensaios

\begin{tabular}{|c|c|c|c|c|c|c|c|c|c|c|c|}
\hline \multirow{3}{*}{$\begin{array}{l}\text { Parâmetros } \\
\text { COV }\left(\mathrm{gDQO}_{\text {total }}(\mathrm{L} \mathrm{d})^{-1}\right.\end{array}$} & \multirow[b]{3}{*}{-} & \multicolumn{8}{|c|}{ Tempo de detenção hidráulica (h) } & \multirow{3}{*}{$\begin{array}{c}\text { CV (\%) } \\
36,7\end{array}$} & \multirow{3}{*}{$\begin{array}{l}\text { Teste } \mathrm{F} \\
22,4^{\star \star}\end{array}$} \\
\hline & & \multicolumn{2}{|c|}{96} & \multicolumn{2}{|c|}{72} & \multicolumn{2}{|c|}{48} & \multicolumn{2}{|c|}{36} & & \\
\hline & & 4,42 & $\mathrm{~b}$ & 5,27 & $\mathrm{~b}$ & 9,33 & a & 11,79 & $\mathrm{a}$ & & \\
\hline \multirow[t]{3}{*}{ NTK (mg L-1) } & Afluente & 987 & $a b$ & 752 & a & 1116 & a & 905 & $a b$ & 34,1 & $3,5^{\star}$ \\
\hline & Efluente & 612 & a & 419 & $b$ & 665 & a & 669 & a & 30,3 & $6,8^{* *}$ \\
\hline & Eficiência (\%) & 37 & a & 39 & a & 38 & a & 31 & $\mathrm{a}$ & 44,4 & $0,6 \mathrm{~ns}$ \\
\hline \multirow[t]{2}{*}{$\mathrm{N}$-am. (mg L-1, } & Afluente & 225 & $a b$ & 164 & $b$ & 258 & a & 274 & a & 29,0 & $8,2^{* \star}$ \\
\hline & Efluente & 452 & $\mathrm{a}$ & 313 & $c$ & 362 & $\mathrm{bc}$ & 426 & $a b$ & 24,0 & $7,0 * \star$ \\
\hline \multirow[t]{3}{*}{$\mathrm{N}$-org. $\left(\mathrm{mg} \mathrm{L}^{-1}\right)$} & Afluente & 763 & a & 588 & $a$ & 858 & a & 631 & $a$ & 41,4 & $2,7 \mathrm{~ns}$ \\
\hline & Efluente & 159 & $\mathrm{bc}$ & 106 & $c$ & 303 & a & 242 & $a b$ & 65,3 & $6,8^{\star *}$ \\
\hline & Eficiência (\%) & 80 & $\mathrm{a}$ & 79 & $\mathrm{a}$ & 65 & $b$ & 59 & $\mathrm{~b}$ & 21,3 & $7,1^{\star \star}$ \\
\hline \multirow[t]{3}{*}{ P-total $\left(\mathrm{mg} \mathrm{L}^{-1}\right)$} & Afluente & 428 & $\mathrm{a}$ & 393 & $\mathrm{a}$ & 488 & $\mathrm{a}$ & 338 & $a$ & 41,5 & $1,9 \mathrm{~ns}$ \\
\hline & Efluente & 335 & $\mathrm{a}$ & 244 & $a b$ & 317 & $a b$ & 221 & $\mathrm{~b}$ & 36,5 & $3,9^{*}$ \\
\hline & Eficiência (\%) & 32 & $\mathrm{a}$ & 36 & $\mathrm{a}$ & 35 & $\mathrm{a}$ & 38 & $\mathrm{a}$ & 49,3 & $0,2 \mathrm{~ns}$ \\
\hline
\end{tabular}

Letras minúsculas diferentes na mesma linha diferem pelo teste de Tukey a 5\%; **: significativo a $1 \%$ de probabilidade $(p<0,01)$; *: significativo a 5\% de probabilidade ( $<<0,05)$; ns: nãosignificativo $(p>0,05)$; p: probabilidade; NTK: nitrogênio total Kjedahl; N-am.: nitrogênio amoniacal; N-org.: nitrogênio orgânico; P-total: fósforo total.

Tabela 6 - Valores médios das concentrações e eficiências médias de remoção de K, Ca, Mg, Na, Cu, Fe, Mn e Zn do afluente e efluentes do ASBR durante os ensaios

\begin{tabular}{|c|c|c|c|c|c|c|c|c|c|c|c|}
\hline \multirow{2}{*}{ Parâmetros } & & \multicolumn{8}{|c|}{ Tempo de detenção hidráulica (h) } & \multirow{2}{*}{ CV (\%) } & \multirow{2}{*}{ Teste F } \\
\hline & & \multicolumn{2}{|c|}{96} & \multicolumn{2}{|c|}{72} & \multicolumn{2}{|c|}{48} & \multicolumn{2}{|c|}{36} & & \\
\hline \multirow{2}{*}{$\mathrm{K}\left(\mathrm{mg} \mathrm{L}^{-1}\right)$} & Afluente & 118 & bc & 59 & c & 152 & $\mathrm{~b}$ & 292 & a & 43,1 & $32,3^{* *}$ \\
\hline & Efluente & 122 & bc & 59 & c & 154 & $\mathrm{~b}$ & 309 & a & 49,9 & $25,3^{\star \star}$ \\
\hline \multirow{3}{*}{$\mathrm{Ca}\left(\mathrm{mg} \mathrm{L}^{-1}\right)$} & Afluente & 657 & $a$ & 677 & a & 269 & $b$ & 171 & $\mathrm{~b}$ & 33,6 & $40,1^{* *}$ \\
\hline & Efluente & 268 & $\mathrm{a}$ & 324 & a & 139 & $\mathrm{~b}$ & 63 & $\mathrm{~b}$ & 36,5 & $33,5^{\star \star}$ \\
\hline & Eficiência (\%) & 58 & a & 52 & $a b$ & 45 & $\mathrm{~b}$ & 63 & a & 21,2 & $5,9 * *$ \\
\hline \multirow{3}{*}{$\mathrm{Mg}\left(\mathrm{mg} \mathrm{L}^{-1}\right)$} & Afluente & 79 & $\mathrm{a}$ & 66 & $a b$ & 46 & c & 57 & c & 30,3 & $7,8^{* *}$ \\
\hline & Efluente & 51 & $\mathrm{a}$ & 42 & $a b$ & 33 & $b$ & 38 & $b$ & 25,2 & $7,5^{\star \star}$ \\
\hline & Eficiência (\%) & 35 & $a$ & 36 & a & 26 & a & 36 & a & 45,0 & $1,3 \mathrm{~ns}$ \\
\hline \multirow{2}{*}{$\mathrm{Na}\left(\mathrm{mg} \mathrm{L}^{-1}\right)$} & Afluente & 48 & $\mathrm{c}$ & 43 & $c$ & 108 & $b$ & 163 & a & 38,7 & 30,0 ** \\
\hline & Efluente & 48 & $\mathrm{c}$ & 31 & $c$ & 85 & $b$ & 143 & $\mathrm{a}$ & 43,6 & $32^{\star \star}$ \\
\hline \multirow{3}{*}{$\mathrm{Cu}\left(\mathrm{mg} \mathrm{L}^{-1}\right)$} & Afluente & 6,8 & $\mathrm{a}$ & 8,2 & $\mathrm{a}$ & 5,4 & $\mathrm{a}$ & 6,6 & $\mathrm{a}$ & 49,1 & $1,7 \mathrm{~ns}$ \\
\hline & Efluente & 1,4 & $\mathrm{a}$ & 1,8 & $\mathrm{a}$ & 2,0 & $\mathrm{a}$ & 2 & a & 70,6 & $0,7 \mathrm{~ns}$ \\
\hline & Eficiência (\%) & 79 & $\mathrm{a}$ & 71 & $\mathrm{a}$ & 61 & $\mathrm{a}$ & 71 & $\mathrm{a}$ & 30,0 & $1,6 \mathrm{~ns}$ \\
\hline \multirow{3}{*}{$\mathrm{Fe}\left(\mathrm{mg} \mathrm{L}^{-1}\right)$} & Afluente & 25,5 & $\mathrm{a}$ & 20,3 & $\mathrm{a}$ & 27,0 & $\mathrm{a}$ & 27,4 & $\mathrm{a}$ & 43,3 & $1,3 \mathrm{~ns}$ \\
\hline & Efluente & 4,5 & $a$ & 6,4 & $a$ & 9,5 & a & 11,4 & $\mathrm{a}$ & 90,6 & $2,5 \mathrm{~ns}$ \\
\hline & Eficiência (\%) & 82 & $\mathrm{a}$ & 68 & $\mathrm{a}$ & 60 & a & 69 & $\mathrm{a}$ & 33,2 & $2,1 \mathrm{~ns}$ \\
\hline \multirow{3}{*}{$\mathrm{Mn}\left(\mathrm{mg} \mathrm{L}^{-1}\right)$} & Afluente & 3,2 & $a$ & 3,7 & $\mathrm{a}$ & 2,9 & $\mathrm{a}$ & 3,6 & $\mathrm{a}$ & 55,7 & $0,5 \mathrm{~ns}$ \\
\hline & Efluente & 1,1 & $\mathrm{a}$ & 1,2 & $\mathrm{a}$ & 1,4 & $\mathrm{a}$ & 1,4 & a & 55,6 & $0,7 \mathrm{~ns}$ \\
\hline & Eficiência (\%) & 62 & $\mathrm{a}$ & 65 & $\mathrm{a}$ & 52 & $\mathrm{a}$ & 63 & $\mathrm{a}$ & 29,5 & $1,2 \mathrm{~ns}$ \\
\hline \multirow{3}{*}{$\mathrm{Zn}\left(\mathrm{mg} \mathrm{L}^{-1}\right)$} & Afluente & 11,1 & $\mathrm{a}$ & 9,1 & $\mathrm{a}$ & 9,2 & a & 7,1 & a & 47,9 & $1,9 \mathrm{~ns}$ \\
\hline & Efluente & 2,0 & $a$ & 2,3 & a & 3,1 & a & 1,9 & a & 78 & $1,2 \mathrm{~ns}$ \\
\hline & Eficiência (\%) & 81 & $\mathrm{a}$ & 71 & $\mathrm{a}$ & 67 & $a$ & 73 & $\mathrm{a}$ & 31,0 & $1,0 \mathrm{~ns}$ \\
\hline
\end{tabular}

Letras minúsculas diferentes na mesma linha diferem pelo teste de Tukey a $5 \%$; ** significativo a $1 \%$ de probabilidade $(p<0,01)$; * significativo a $5 \%$ de probabilidade ( $p<0,05)$; ns: nãosignificativo $(p>0,05)$; $p$ : probabilidade. 
Tabela 7 - Valores médios e coeficientes de variação (CV) das concentrações de sólidos totais (ST) e sólidos voláteis (SV) do lodo sedimentado no ASBR, das taxas de carregamento orgânico do lodo (TCL) e do tempo de retenção de sólidos (TRS), durante os ensaios

\begin{tabular}{|c|c|c|c|c|c|c|c|c|c|c|}
\hline \multirow{3}{*}{$\begin{array}{l}\text { Parâmetros } \\
\text { ST }\left(\mathrm{g} \mathrm{L}^{-1}\right)\end{array}$} & \multicolumn{10}{|c|}{ Tempo de detenção hidráulica (h) } \\
\hline & \multicolumn{2}{|c|}{96} & \multicolumn{2}{|c|}{72} & \multicolumn{2}{|c|}{48} & \multicolumn{2}{|c|}{36} & \multirow{2}{*}{$\begin{array}{c}\text { CV (\%) } \\
61\end{array}$} & \multirow{2}{*}{$\begin{array}{l}\text { Teste F } \\
2,1 \text { ns }\end{array}$} \\
\hline & 3,5 & a & 6,8 & a & 4,8 & a & 3,5 & a & & \\
\hline$S V\left(g L^{-1}\right)$ & 2,4 & a & 4,0 & a & 3,5 & a & 5,3 & a & 73 & $1,4 \mathrm{~ns}$ \\
\hline SV/ST & 0,69 & $a b$ & 0,58 & $\mathrm{~b}$ & 0,74 & a & 0,70 & $a b$ & 15 & $3,5^{\star}$ \\
\hline TCL (gDQO (g SV d) $\left.)^{-1}\right)$ & 1,9 & bc & 1,7 & c & 3,5 & a & 2,7 & $a b$ & 42 & $9,3^{\star \star}$ \\
\hline TRS (d) & 12,0 & $a$ & 9,4 & $a b$ & 3,0 & $\mathrm{~b}$ & 5,1 & $a b$ & 63 & $5,2^{\star *}$ \\
\hline
\end{tabular}

Letras minúsculas diferentes na mesma linha diferem pelo teste de Tukey a $5 \%$; ** significativo a $1 \%$ de probabilidade; * significativo a $5 \%$ de probabilidade; ns: não- significativo.

As concentrações médias de Fe no afluente variaram de 20,34 a 27,40 $\mathrm{mg} \mathrm{L}^{-1}$; de zinco (Zn), de 7,13 a 11,12 $\mathrm{mg} \mathrm{L}^{-1}$; de cobre (Cu), de 5,40 a 8,26 $\mathrm{mg} \mathrm{L}^{-1}$; de manganês (Mn), de 2,94 a 3,5 $\mathrm{mg} \mathrm{L}^{-1}$. As concentrações médias de ferro (Fe) no efluente do ASBR durante os quatro ensaios variaram de 4,5 a 11,1 $\mathrm{mg} \mathrm{L}^{-1}$; de $\mathrm{Zn}$, de 1,9 a 3,0 $\mathrm{mg} \mathrm{L}^{-1}$; de $\mathrm{Cu}$, de 1,4 a 2,0 $\mathrm{mg} \mathrm{L}^{-1}$; de $\mathrm{Mn}$, de 1,1 a 1,4 $\mathrm{mg} \mathrm{L}^{-1}$ (Tabela 6). As concentrações de Fe e Zn no efluente do ASBR, em todos os ensaios, foram menores que valores máximos contidos no padrão de lançamento de efluentes estabelecido na resolução $n^{\circ} 357$ do Conama (BRASIL, 2005), que é de $15 \mathrm{mg} \mathrm{L}^{-1}$ para o Fe e de $5 \mathrm{mg}$ $\mathrm{L}^{-1}$ para o $\mathrm{Zn}$.

Não foram observadas diferenças significativas entre as eficiências de remoção de NTK, P-total, Mg, Cu, Fe, Mn e Zn com a variação das COV nos ensaios ( $p>0,05)$. Resultados semelhantes para o P-total e o NTK, com remoções de 13\% para o P-total e de $20 \%$ para o NTK, foram observados por Nedegwa et al (2008), tratando águas residuárias de suinocultura em um reator ASBR com volume de $12 \mathrm{~L}$ e com COV variando de 0,4 a 1,2 g DQO $(\mathrm{L} \mathrm{d})^{-1}$. Os autores atribuíram esse resultado à capacidade do sistema de tratamento anaeróbio de preservar os nutrientes em virtude da pequena imobilização de nitrogênio e fósforo no lodo anaeróbio.

Observaram-se eficiências médias de remoção de 60 a $82 \%$ para o Fe, de 67 a $81 \%$ para o Zn, de 61 a $79 \%$ para o Cu e de 52 a $65 \%$ para o Mn. Masse et al (2007) verificaram eficiências médias de remoção menores (de $15 \%$ para o Fe; $41,5 \%$ para o $\mathrm{Cu} ; 21 \%$ para o Mn e 18,4 \% para o Zn) em um ASBR tratando águas residuárias de suinocultura mesmo com COV menor (de 0,14 g DQO $\left.\mathrm{total}(\mathrm{L} \mathrm{d})^{-1}\right)$ do que as aplicadas nos ensaios 1 e 4 .

Os valores médios das concentrações de sólidos totais (ST) e sólidos voláteis (SV) do lodo sedimentado no ASBR foram baixos (Tabela 7), comparando-se com os valores obtidos para lodo de reatores anaeróbios, UASB e compartimentado (ABR), na faixa de 4 a $79 \mathrm{~g} \mathrm{~L}^{-1}$ de SV (SANTANA, 2004; FERNANDES, 2004), tratando águas residuárias de suinocultura com SST de 2 a $7 \mathrm{~g} \mathrm{~L}^{-1}$ e COV similares às utilizadas nos ensaios 1 a 4

Os valores médios da relação SV/ST para o lodo do Rl foram de 0,69;0,58; 0,74 e 0,70 durante os ensaios $1,2,3$ e 4, respectivamente (Tabela 7). As altas relações SV/ST evidenciam a predominância de matéria orgânica no lodo, e consequentemente da presença de microrganismos, considerando-se a produção de metano obtida (de 0,50 a 0,64 L (L reator d) $)^{-1}$ ). (OLIVEIRA et al, 1997). Além disso, segundo a resolução no 375 de 29 de agosto de 2006, do Conama (BRASIL, 2006), para fins de utilização agrícola, o lodo de esgoto ou produto derivado será considerado estável se a relação entre sólidos voláteis e sólidos totais for inferior a 0,70 - o que não foi obtido somente com TDH de 48 horas, em virtude da ocorrência de temperaturas médias na faixa psicrofílica, provocando o decréscimo da atividade da microbiota do lodo.

Os valores médios das taxas de carregamento orgânico no lodo (TCL) do ASBR foram de 1,9; 1,7; 3,5 e 2,7 g DQO $_{\text {total }}$ (g $\left.\mathrm{SV}_{\text {lodo }} \mathrm{d}\right)^{-1}$ com os TDH de 96, 72, 48 e 36 horas, respectivamente (Tabela 7). A TCL, durante o regime permanente e de acordo com o tipo de água residuária a ser tratada, pode atingir valores em torno de 2,0 kg DQO $\mathrm{kgtal}\left(\mathrm{kg} \mathrm{SV}_{\text {lodo }} \mathrm{d}\right)^{-1}$ em reatores anaeróbios (CHERNICHARO, 1997).

A aplicação de valores médios de TCL de até 2,7 g DQO total $_{(\mathrm{g}}$ $\left.\mathrm{SV}_{\text {lodo }} \mathrm{d}\right)^{-1}$ no ASBR com TDH de 96, 72, 48 e 36 horas e temperaturas médias de aproximadamente $23^{\circ} \mathrm{C}$, não foram limitantes para a obtenção de eficiências médias de remoção de $\mathrm{DQO}_{\text {total }}$ e SSV acima de $68 \%$ e produção volumétrica de metano de 0,50 a 0,64 $\mathrm{L} \mathrm{CH}_{4}$ (L reator $\mathrm{d})^{-1}$. Para o TDH de 48 horas, com temperatura média em tor-

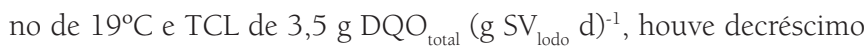
significativo nas eficiências de remoção de $\mathrm{DQO}_{\text {total }}$ e SSV, que foram em torno de 55\%, e da produção volumétrica de metano de 0,53 L $\mathrm{CH}_{4}$ (L reator $\left.\mathrm{d}\right)^{-1}$.

O tempo de retenção de sólidos (TRS) no ASBR foi de 12,0; 9,4; 3,0 e 5,1 d, nos ensaios 1, 2, 3 e 4, respectivamente. Com o aumento da COV nos ensaios 1, 2 e 4, houve diminuição do TRS no ASBR. De acordo com Ndon E Dague (1997), o aumento da COV provoca maior produção de microrganismos dispersos e consequentemente o decréscimo do TRS.

Segundo Zhang et al (1997), com o aumento da COV, o decréscimo do TRS ocorre em virtude do aumento de sólidos suspensos no reator, que pode diminuir a sedimentação do lodo e consequentemente 
Tabela 8 - Valores médios das concentrações de N, P, K, Ca, Mg, Na, Cu, Fe, Mn e Zn no lodo sedimentado coletado no ASBR ao final dos ensaios

\begin{tabular}{lcccc|}
\hline \multirow{2}{*}{$\begin{array}{l}\text { Nutrientes } \\
\text { (mg/kg base seca) }\end{array}$} & \multicolumn{4}{c}{ Tempo de detenção hidráulica (h) } \\
\cline { 2 - 5 } & 96 & 72 & 48 & 36 \\
\hline $\mathrm{N}$ & 64.400 & 30.610 & 39.900 & 62.400 \\
\hline $\mathrm{P}$ & 4.470 & 9.870 & 1.590 & 3.481 \\
\hline $\mathrm{Ca}$ & 6.760 & 5.970 & 4.040 & 1.180 \\
\hline $\mathrm{Mg}$ & 440 & 370 & 170 & 347 \\
\hline $\mathrm{Na}$ & 130 & 120 & 40 & 450 \\
\hline $\mathrm{K}$ & 290 & 120 & 160 & 190 \\
\hline $\mathrm{Fe}$ & 8.040 & 8.700 & 7.410 & 6.180 \\
\hline $\mathrm{Cu}$ & 950 & 490 & 550 & 1.250 \\
\hline $\mathrm{Zn}$ & 1.070 & 600 & 630 & 1.370 \\
\hline $\mathrm{Mn}$ & 310 & 256 & 180 & 510 \\
\hline
\end{tabular}

aumenta o arraste de lodo com o efluente. Schmit e Dague (1993), tratando águas residuárias de suinocultura em ASBR e aplicando COV de 3,31 a 5,37 g SV (L d)-1, encontraram TRS de 18,04 a 8,52 dias. Os autores observaram que a diminuição da COV e o aumento do TDH resultaram em maiores TRS, o que também foi observado neste trabalho.

Com as baixas temperaturas, a viscosidade do fluído no reator aumenta, diminuindo a velocidade de sedimentação do lodo, especialmente dos microrganismos dispersos (NDON; DAGUE, 1997), o que pode ter acorrido no ensaio 3, com TRS de 3,0 dias e temperaturas médias de $19,2{ }^{\circ} \mathrm{C}$.

O TRS mínimo para o processo metanogênico é de aproximadamente 10 dias para a temperatura de $35^{\circ} \mathrm{C}$ (NDON; DAGUE, 1997). Nos ensaios 3 e 4 , observaram-se valores abaixo do recomendado. O TRS de 5,1 d no ASBR, observado no ensaio 4, não foi limitante para a obtenção de altas eficiências médias de remoção de $\mathrm{DQO}_{\text {total }}$ e SST de 82 e $80 \%$, respectivamente, e produção de metano de $0,5 \mathrm{~L}$ (L reator $\mathrm{d})^{-1}$.

As concentrações médias de $\mathrm{N}$ e $\mathrm{P}$ no lodo do ASBR, durante os ensaios, variaram de 30.610 a 64.400 e de 9.870 a 1.590 mg (kg base seca $^{-1}$, respectivamente (Tabela 8).

O N, P, Fe e Ca foram os nutrientes encontrados em maiores concentrações no lodo no ASBR. Segundo Oliveira et al (1997), os mecanismos de precipitação no lodo podem ser a formação da estrovita $\left(\mathrm{MgNH}_{4} \mathrm{PO}_{4}\right)$ e vivianita $\left(\mathrm{Fe}_{3} \mathrm{PO}_{4} \cdot 8 \mathrm{H}_{2} \mathrm{O}\right)$, além da hidroxiapatita
$\left(\mathrm{CaHPO}_{4}\right)$, as quais podem representar as formas de remoção de nutrientes com inclusão no lodo, principalmente N, P, Fe e Ca, das águas residuárias, como as provenientes da suinocultura.

As concentrações médias de Cu e Zn foram de 490 a 1.250; 600 a $1370 \mathrm{mg}$ (kg base seca) ${ }^{-1}$, respectivamente, no lodo do ASBR (Tabela 8). Segundo a resolução $n^{\circ} 375$ do Conama, o lodo de esgoto e de produtos derivados, para o uso agrícola, deve respeitar os limites máximos de concentração de $\mathrm{Cu}$ e Zn de 1.500 e $2.800 \mathrm{mg}$ (kg base $\mathrm{seca}^{-1}$, respectivamente. As concentrações de $\mathrm{Mg}$, $\mathrm{Na}$ e K variaram de 40 a $450 \mathrm{mg}(\mathrm{kg} \text { base seca) })^{-1}$ de lodo.

\section{Conclusões}

Com a utilização do ASBR, foi possível obter eficiências de remoção de DQO e sólidos suspensos de 50 a 87\%, de NTK, P, e Mg de 30 a 39\%, e de N-org, Ca, Cu, Fe, Mn e Zn de 50 a 82\%, com produções de metano de 0,50 a $0,64 \mathrm{~L} \mathrm{CH}_{4}$ (L reator d) ${ }^{-1}$, para COV de 4,4 a 11,8 $\mathrm{g} \mathrm{DQO}_{\text {total }}(\mathrm{L} \mathrm{d})^{-1}$, indicando que o seu emprego com TDH de até 36 horas pode ser viável no tratamento de águas residuárias de suinocultura, principalmente em virtude da vantagem da operação intermitente. Com valores médios de temperatura do ar abaixo de $20^{\circ} \mathrm{C}$, a obtenção de eficiências de remoção de $\mathrm{DQO}_{\text {total }}$ e sólidos suspensos acima de $60 \%$ exigirá a utilização de TDH maiores que 48 horas.

Os valores médios da relação SV/ST do lodo proveniente do ASBR foi de 0,58 a 0,74 e evidenciam a predominância de matéria orgânica no lodo e consequentemente da presença de microrganismos, considerando-se a produção de metano obtida (de 0,50 a 0,64 $\left.\mathrm{L}(\mathrm{L} \text { reator } \mathrm{d})^{-1}\right)$, e com características de estabilidade para o uso agrícola no solo (SV/ST inferior a 0,70), quando for descartado o excesso de lodo. As médias de $\mathrm{Cu}$ e Zn no lodo do ASBR de 490 a 1.250 e 600 a $1.370 \mathrm{mg}$ ( $\mathrm{kg}$ base seca) $)^{-1}$, tomando-se por base os limites estabelecidos na resolução no 375 do Conama, é possível o uso agrícola do lodo.

\section{Agradecimentos}

À Fundação de Amparo à Pesquisa do Estado de São Paulo (Fapesp), à Coordenação de Aperfeiçoamento de Pessoal de Nível Superior (Capes) e à Tigre S/A Tubos e Conexões, pelo apoio financeiro. 


\section{Referências}

AHN, J.H et al. The effect of calcium on the anaerobic digestion treating swine wastewater. Biochemical Engineering Journal, v. 30, p. 33-60, 2006.

APHA, AWWA, WPCF. Standard methods for the examination of water and wastewater. 20th ed. Washington, DC: APHA, 1998.

BRASIL. Ministério do Meio Ambiente. Conselho Nacional do meio Ambiente (Conama). Resolução n 357, de 17 de março de 2005. Dispõe sobre a classificação dos corpos de águas e diretrizes ambientais para o seu enquadramento, bem como estabelece as condições e padrões de lançamento de efluentes, e dá outras providências. Brasília, DF: Ministério do Meio Ambiente, 2005.

Ministério do Meio Ambiente. Conselho Nacional do meio Ambiente (Conama). Resolução no 375, de 29 de agosto de 2006. Define critérios e procedimentos, para o uso agrícola de lodos de esgoto gerados em estações de tratamento de esgoto sanitário e seus produtos derivados, e dá outras providências. Brasília, DF: Ministério do Meio Ambiente, 2006

CHERNICHARO, C.A.L.. Reatores anaeróbios: princípios do tratamento biológico de águas residuárias. Belo Horizonte: Polytécnica, 1997.

COSTA, R.H.R.; MEDRI, W. Modelling and optimisation of stabilisation ponds system for the treatment of swine wastes: organic matter evaluation. Brazilian Archives of Biology and Technology, Curitiba, v. 45, n. 3, p. 385-392, 2002

DAGUE, R.R; HABBEN, C.E.; PIDAPARTI, S.R. Initial studies on the anaerobic sequencing batch reactor. Water Science and Technology, Oxford, v. 26, n. 9-11, p. 2429-2432, 1992.

DILALLO, R.; ALBERTSON, O.E. Volatile acids by direct titration. Journal Water Pollution Control Federation, Alexandria, v. 33, n. 4, p. 356-365, 1961

FERNANDES, G.F.R; OLIVEIRA, R.A. Desempenho de processo anaeróbio em dois estágios (reator compartimentado seguido de reator UASB) para tratamento de águas residuárias de suinocultura, Engenharia Agrícola, Jaboticabal, v. 26, n. 1, p. 243-256, jan./jun. 2006.

FERNANDES, G.F.R. Desempenho de processo em dois estágios (reator compartimentado seguido de reator UASB) para tratamento de águas residuárias de suinocultura. 2004. 136. Dissertação (Mestrado em Microbiologia Agropecuária) - Faculdade de Ciências Agrárias e Veterinárias da Unesp, Jaboticabal, 2004

INSTITUTO BRASILEIRO DE GEOGRAFIA E ESTATÍSTICA, (IBGE). [Online]. Banco de dados agregados. Sistema IBGE de Recuperação Automática, SIDRA. Disponível em: <http://sidra.ibge.gov.br>. Acesso em: 15 mar. 2008

LUDKE, J.V.; LUDKE, M.C.M.M. Preservação ambiental. Suinocultura Industrial, São Paulo, v. 25, n. 2, p. 10-14, 2002.

MASSÉ, DI.; CROTEAU, F; MASSE, L. The fate of crop nutrients during digestion of swine manure in psychrophilic anaerobic sequencing batch reactors. Bioresource Technology, Barking, v. 98, n. 15, p. 2819-2823, 2007

MASSÉ, D.I. et al. Potential for the psychrophilic anaerobic treatment of swine manure using a sequencing batch reactor. Canadian Agricultural Engineering, v. 1, n. 39, p. 25-33, 1997

MASSÉ, D.I.; MASSE, L.; CROTEAU, F. The effect of temperature fluctuations on psychrophilic anaerobic sequencing batch reactors treating swine manure. Bioresource Technology, Barking, v. 89, n. 1, p. $57-62,2003$

MATA-ALVAREZ, J. (Ed.) Biomethanization of the organic fraction of municipal wastes. London: IWA Publishing, 2003.

METCALF, E. Wastewater engineering: treatment, disposal and reuse. 3rd ed. New York: McGraw-Hill, 1991.

MIRANDA, C.R. de. Avaliação de estratégias para sustentabilidade da suinocultura. 2005. 264. Tese (Doutorado em Engenharia Ambiental) Universidade Federal de Santa Catarina, Florianópolis, 2005.

NDEGWA, P.M. et al. Effects of cycle-frequency and temperature on the performance of anaerobic sequencing batch reactors (ASBRS) treating swine waste. Bioresource Tecnology, v. 99, n. 6, p. 1972-1980, 2008

NDON, U.J.; DAGUE, R.R. Effects of temperature and hydraulic retention time on anaerobic sequencing batch reactor in the treatment of low-strength wastewater. Water Research, Oxford, v. 31, n. 10, p. 2455-2466, 1997.

OLIVEIRA, R.A. ; VAZOLLER, R.F.; FORESTI, E. Sludge bed characteristics of UASB reactors: growth, activity, microbial structure, and chemical composition of granules. In: $8^{\text {th }}$ INTERNATIONAL CONFERENCE ON ANAEROBIC DIGESTION, Proceedings... Sendai, Japan: IAWQ, p. 524$531,1997$.

PINHEIRO, D.M. et al. Fluidized ASBR treating synthetic wastewater: Effect of recirculation velocity. Chemical Engineering and Processing, $v$. 47, n. 2, p. 184-191, 2008.

RODRIGUES, R.S.; SELBACH, P.A. Redução de carga poluidora em lodo de suinocultura através de filtração. Revista Brasileira de Agrociência, Pelotas, v. 9, n. 4, p. 407-411, 2003

SANTANA, A.M. Atividade da microbiota e desempenho de reatores anaeróbios de fluxo ascendente com manta de lodo (UASB) em dois estágios tratando águas residuárias de suinocultura. 2004. 113 f. Dissertação (Mestrado em Microbiologia Agropecuária) - Faculdade de Ciências Agrárias e Veterinárias da Unesp, Jaboticabal, 2004.

SANTANA, A.M. OLIVEIRA, R.A. Desempenho de reatores anaeróbios de fluxo ascendente com manta de lodo em dois estágios tratando águas residuárias de suinocultura. Engenharia Agrícola, Jaboticabal, v. 25, n. 3, p. $817-830,2005$

SCHMIT, C.G.; DAGUE R.R. Anaerobic sequencing batch reactor treatment of swine wastes at $20^{\circ} \mathrm{C}, 25^{\circ} \mathrm{C}$, and $35^{\circ} \mathrm{C}$. In: 48th PURDUE UNIVESITY Industrial Waste Conference, Proceedings... Chelsea: Ann Arbor Press, p. 541-549, 1993. 
SHIZAS, I.; BAGLEY, D.M. Improving anaerobic sequencing batch reactor performance by modifying operational parameters. Water Research, Oxford, v. 36, n. 1, p. 363-367, 2002.

WORD HEALTH ORGANIZATION (WHO). Guidelines for the use of wastewater in agriculture and aquaculture. Technical Report Series, 778. Genebra: WHO, 1989.
WORD HEALTH ORGANIZATION (WHO). Guidelines for the safe use of wastewater, excreta and greywater - policy and regulatory aspects. v. 1. Genebra: WHO, 2006.

ZHANG, R.H. et al. Anaerobic treatment of swine waste by the anaerobic sequencing batch reactor. Transactions of the ASAE, St. Joseph, v. 40 n. 3, p. 761-767, 1997. 